\title{
Design of ARM based Enhanced Event Data Recorder \& Evidence Collecting System
}

\author{
Nitin P. Sirsikar, Prof. Pankaj H. Chandankhede \\ ${ }^{I}$ Student, M.Tech (Communication Engineering) Electronics and Telecommunication Engineering \\ G. H. Raisoni College of Engineering Nagpur, India \\ ${ }^{2}$ Research Scholar Electronics and Telecommunication Engineering G. H. Raisoni College of Engineering \\ Nagpur, India
}

\begin{abstract}
Design of ARM based Enhanced Event Data Recorder \& Evidence Collecting System using GPS and different sensors. This proposed design provides information related to vehicle speed and position on real time basis and status about temperature of engine, front obstacle detection and alcohol detection. This information collected by ARM7 processors using module and sensors which are connected to the processor and also gives all the collected data to the monitoring station. The system can record the data in real time which can help people to analyze the fault protection at extreme conditions design in Event Data Recorder which displays the messages on LCD. It can also be used to analyze the accident easily and to settle many disputes related to car accident such as investigation, vehicle maintenance, insurance settlement, crash litigation, driver performance.
\end{abstract}

Index Terms: ARM7 processor, event data recorder, GPS, GSM module

\section{Introduction}

In 2008, Road Traffic Injuries (RTI) ranked fourth among the leading causes of death in the world. Around 1.3 million people die every year on the world's roads; $20-50$ million people suffer non-fatal injuries with many sustaining a disability as a result of their injury. As the population increases the number of vehicles also increases on the road and highways, these results in more accident and public need to get immediately. The proposed module provides information about the accident to the monitor station, to the hospital and police station. To improve the level of supervision and management for hard and heavy traffic vehicles, trucks carrying coal it is important to develop transport vehicles remote monitoring module Like, Black Box of airplane, event data recorder used to record information related to accidents. Car Event Data Recorder records driving data, collision data and position data before and after the accidents occurred so that it can be used to analyze the accident and also used to reconstruct what happened before an accident and through which improve vehicle design, roadway design, and emergency medical service by automakers and government.

The project is about the composition and function of the car black box. The system can record the driving data in real time which can help people to analyze the fault protection at extreme conditions design in Event Data Recorder which displays messages on LCD to avoid further accident event. The Car Black Box can receive real time data including speed, position and status about the all sensors. The design consists of ARM processor, A/D convert module, storage unit, input-output interface, GPIO, UART and different sensing modules. Collecting, processing and storing the multiple signals at the same time which need real time processor having ability to read and write with high speed. SD card is required for large capacity and long term data storage to maintain data without any damage and lost. This event data recorder provides the fault protection message on LCD. 


\section{System Description}

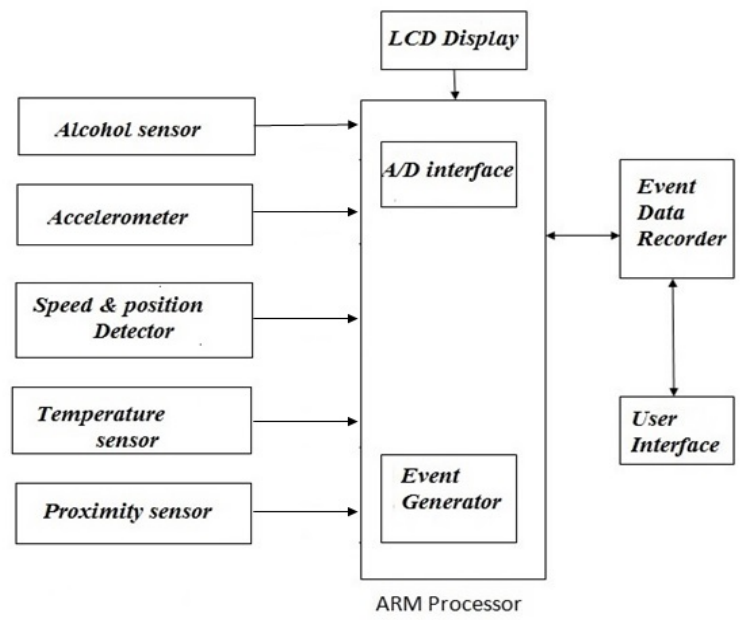

Fig.1 Block diagram of event data recorder

\section{Data Collection}

In data collection step, collecting various driving signals and status information of the vehicle in the Event Data Recorder.

\section{Data Processing}

Data processing is used to receive data information from the data collection unit. The software of the system runs in this unit, the application code written in embedded C. The storage unit includes SD card.

\section{Human-computer Interaction Layer}

The human computer interaction layer is the interactive platform for the human beings and the Vehicular Black Box. The data information of a car will be displayed on the screen of personal computer after being received.

\section{A. Event Data Recorder}

\section{System Model}

In Event Data Recorder, Crash data retrieval (CDR) system and Sensing and Diagnostic Module (SDM) two systems can be used. Crash data retrieval (CDR) system used to download pre and post crash data from the vehicles airbag module to a computer. Sensing and Diagnostic Module (SDM) is used for GM EDR which has capability to store a description of both the crash and the pre-crash phase of a traffic collision.

\section{- Pre-Crash Data}

New version of the GM EDR can store up to 5 seconds of pre-crash data. In data elements, includes vehicle speed, engine throttle position, engine revolutions per minute and break status versus time for 5 seconds preceding the time for airbag control module. These data elements provide a record of the actions taken by the driver just prior to the crash.

\section{- Data Elements Recorded during the Crash}

During the crash occurred, the most valuable data element stored in the EDR is in the form of longitudinal changes in velocity versus time history of the vehicle. Changes in velocity are referred to as deltaV. In GM EDRs longitudinal delta-V is recorded every 10 milliseconds for up to 300 milliseconds in older version of EDR and in new version of EDR it is up to 150 milliseconds. Lateral delta-V is not recorded. Figure 2 shows the longitudinal delta-V vs. time recorded by an EDR. 


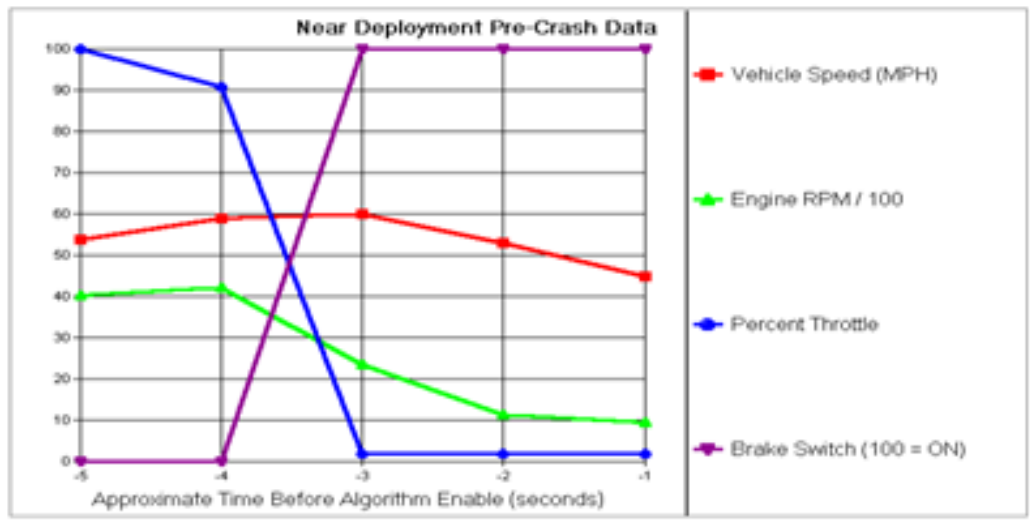

Fig 2: Example of GM EDR pre crash information

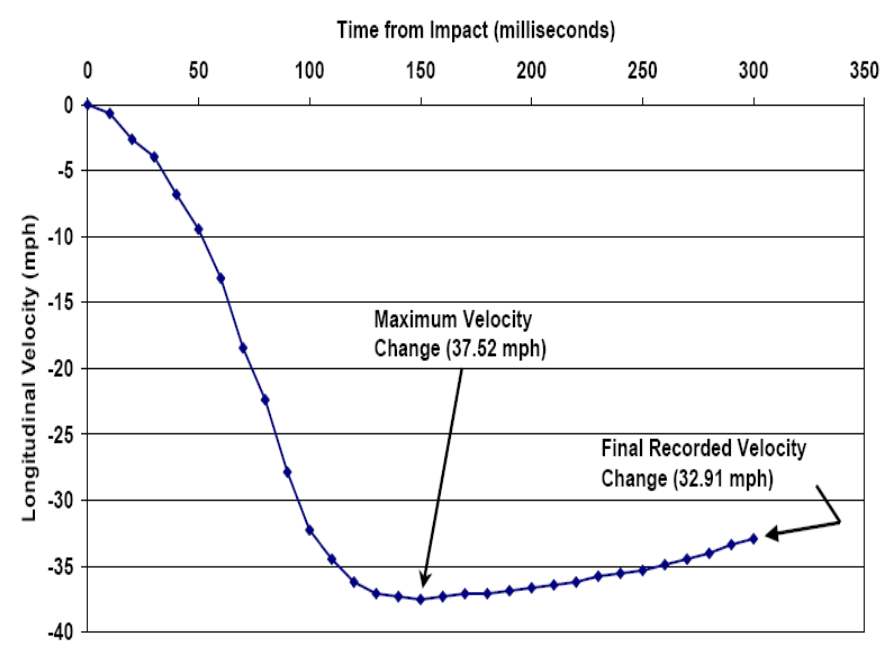

Fig 3: GM EDR record for longitudinal velocity vs. time

\section{- Storing multiple car crash}

Many crashes are composed with several impact events. Two events can be stored in EDRs associated with a crash. The EDRs can store 3 different types of events non-deployment event, a deployment event and a deployment-level event. A non- deployment is defined as a crash of very low a severity to warrant deploying the airbag. A deployment event is an impact on which the air bag was deployed. A deployment-level event is an impact of severity that the airbag would have been deployed.

\section{B. ARM7 processor}

The conventional 8 and 16 bit Microcontroller has its deficiencies when compared with 32bit microprocessor. Philips LPC2148 which is based on 32 bit ARM7 TDMI core supporting real time simulation. When ARM processor combined with RTOS with timing constraint can be realized for the data acquisition and transmission of data with high precision.

\section{GPS MODULE}

Global Position System (GPS) is a space-based satellite navigation that provides location and time information. This GPS receiver gives data output in form of standard National marine electronics association (NMEA) format. The data can be collected in GPS receiver in NMEA format is received on ARM processor using UART protocol which contains information about Vehicle position (longitude, latitude) and speed. This information can be check on HyperTerminal of a computer using USB to serial convertor. GPS Receivers commonly used in most Vehicle tracking systems can only receive data from GPS Satellites. They cannot communicate back with GPS or any other wireless devices. A system based on GPS can only calculate its location; normally use GSM-GPRS Cellular networks connectivity using additional GSM modem/module. 


\section{Data Storage}

The related Event sensor data is stored into the SD card. The SD card is memory design to meet security, capacity and performance. It includes protection mechanism and supports easily interface allowing a PC to be connected without special devices. All PC stored data can later recovered using PC equipped.SD implemented using serial peripheral interface protocol supported by LPC2148 ARM7 processor.

\section{E. Real Time Monitor}

Real Monitor is a configurable software module which is developed by ARM inc. information, taken from the ARM processor which is applied to a specific configuration of Real Monitor software programmed in the on-chip ROM boot memory of this device which allows user to establish a debug session to a currently running system.

The system flow takes place in the following manner:

- The System is started.

- The system senses the parameter from various parts of the vehicle.

- Then sends the collected information to the ADC.

- Then ARM processes the data and takes immediate steps.

- If sensors parameters exceed their limit then ARM LPC2148 send commands to the relay to stop the engine.

- If for a crash or a accident, it sends the data over GPS and GSM of the user.

- Or if the parameter does not exceed the limit it will be continued.

\section{Circuit Description}

The LPC2148 is an ARM processor which is the main part of the system. LPC2148 has two UART ports and we are using port 1. GPS module is connected to UART port 1 through RS232 and the LPC kit refresh in every 10 seconds. Arduino circuit, two sensors, GPS modem and GSM module are connected to the LPC2148.

LPC 2148 consists of two switches, one is used for adjusting the temperature values which are used for the temperature rotating or flipped. $+3.3 \mathrm{~V}$ power supply needed to LPC2148 Tyro board. Now the screen shows the current temperature readings. By Bringing a Hot soldering iron tip near the LM35's pins and keeping it 1 or $2 \mathrm{~mm}$ away, the screen shows the rise in the temperature. Keep it there until temperature rise to 100 degrees Celsius, and then remove the iron from LM35'S pin. The data about the temperature changes of the engine as well as the cabin is continuously monitored and then sent to the processor by this sensor LM 35 . If in any case the temperature of engine or cabin exceeds desire value then system alerts to driver and the driver can take immediate steps for the further actions.

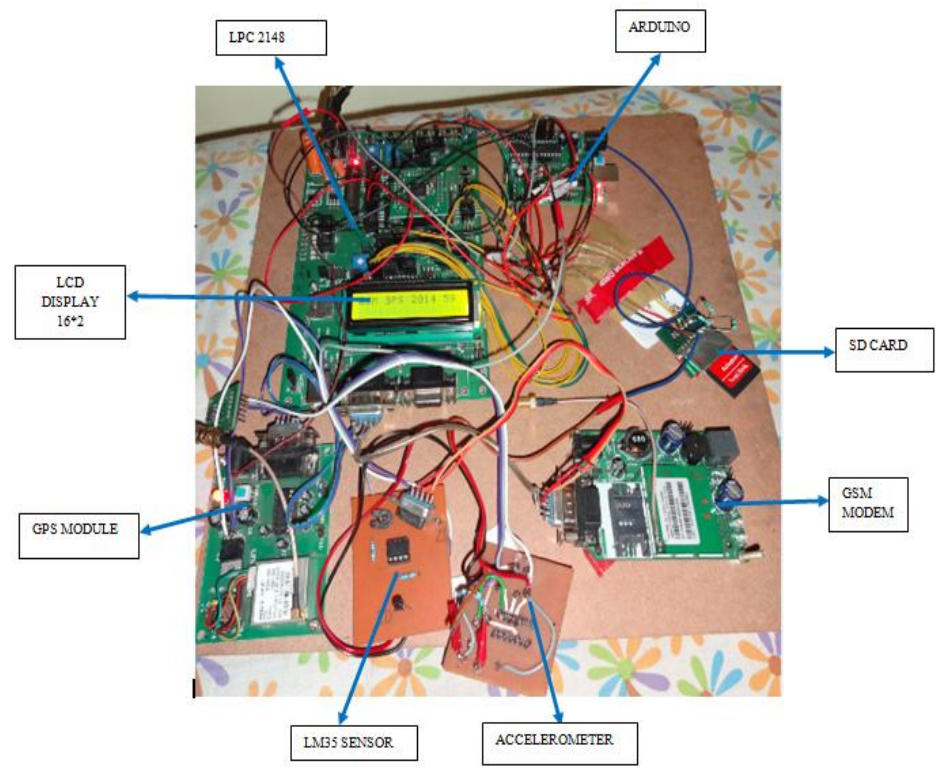

Fig.4. Circuit Diagram

The Accelerometer is connected to the voltage regulator. For example there is dash or the car meets with an accident, the accelerometer vibrates and logic 0 is given out. This logic 0 is the indication for the circuit 
that the car has met with an accident and thus it sends the data of car's location to the users provided in the AT commands. Thus logic 0 is an indication to send the signals. There is a time lapse between the car dash and the sending of message; this is indicated by the LED on the LPC2148 circuit.

The data is stored in the SD card. The format in which the data is saved in the recorder is not the txt format and also is not arranged properly manner. Thus for arranging and changing the format, we require a circuit called Arduino. This circuit changes the format and then also arranges the data in a sequence. This data is continuously stored in the .txt format in a Datalog file in format of latitude then longitude and lastly temperature. Initially, when there is no data or the system is just started, the values stored are the garbage values. After initiating the values, the actual reading of the temperature is stored in the file "Datalog". The temperature is shown in the Degree Celsius. Only a single file is updated here, i.e. no need to develop another file again.

These stages are repeated and the number of times to get the updating of the temperatures and the other factors in a continuous manner. The old data gets saved and the process is repeated again and again. For storing the all type of large data, SD card storage capacity will also be requires in large amount.

\section{Result}

The project "vehicular black box" which would help to analysis the root cause of accident with the help of data from various sensors connected inside the vehicle that are explained below as per the following parameters :-

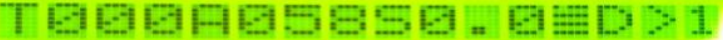

Fig.5: Result of Alcohol detection

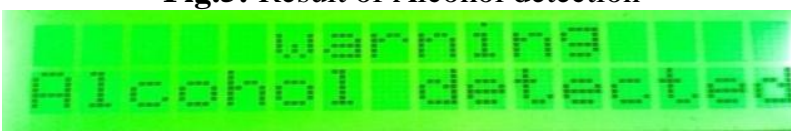

Fig.6: Warning after Alcohol detection

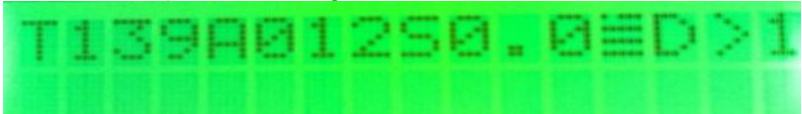

Fig.7: Result for Exceed temperature

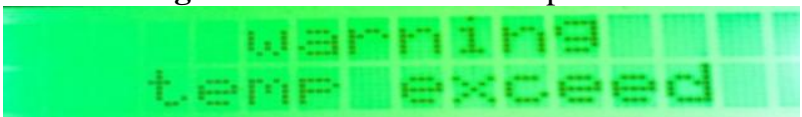

Fig.8: Warning after Temperature exceed

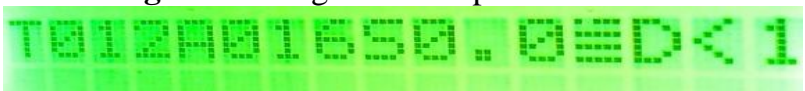

Fig.9: Result for distance crossed at less than 1 feet

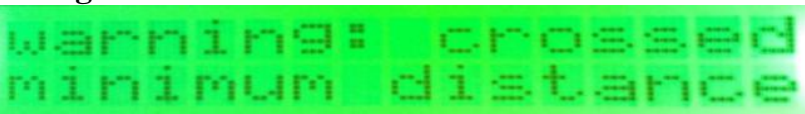

Fig.10: Warning after Temperature exceed

- When the temperature increases over a particular value (100 Degree Celsius), the message is sent to the user and at the same time the data is stored in the SD card given in the circuit.

- These are the most important parameters for providing the output.

- The coordinates shown are the exact coordinates at which the system is present. Thus the result is achieved. Thus it can be of great help.

- If any harm is provided to the system, the data is sent to the user and also saved in the memory card. The file format of the text stored is .txt type by using Arduino circuit.

The GSM sends the coordinates to the user whose number is saved in the SIM provided in the GSM kit. The number is saved as by using the AT commands. 


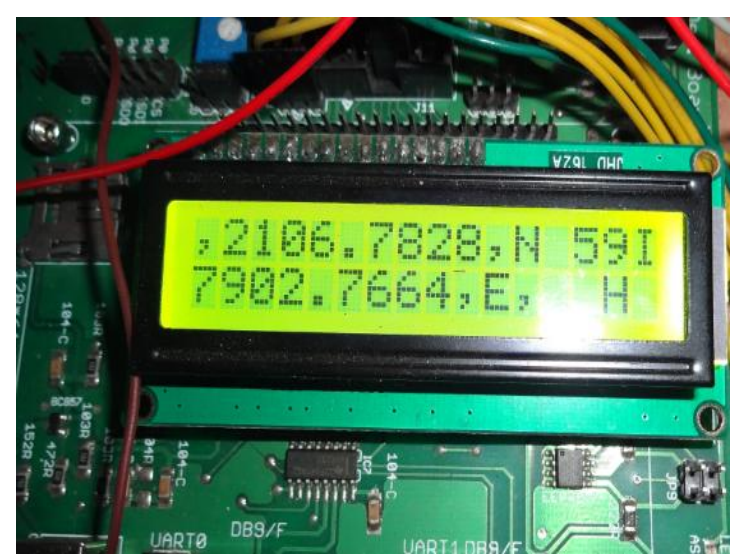

Figure 11: Output of GPS on LCD Screen

It is thus possible to help spot the accident positions. It thus helps to lessen the number of deaths caused due to not knowing the locations of the accidents.

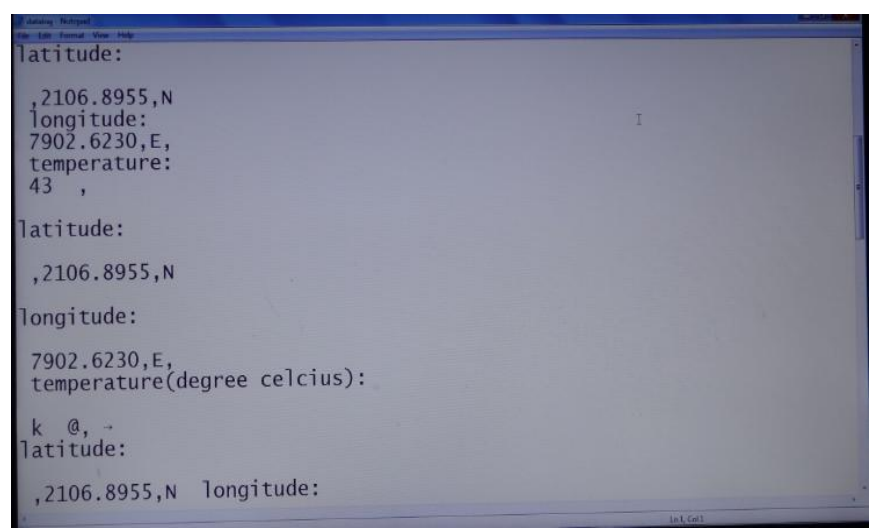

Figure 12: Data stored in .txt format

\section{For Personal vehicle}

\section{Application}

If any accident had occurred to a vehicle fitted with black box then immediate help can be provided to the victimized car on receiving SMS.

\section{Insurance companies}

Most of the time of accident is false so insurance companies can implement this car black box in the insured vehicle and as a data before and at the time of accident is locked into black box. The insurance company can easily analyze the data recorded, and they can find out whether the actually accident occurred or not.

\section{Military purpose}

Military vehicles carry ammunition from one place to other for example in Kashmir military vehicles can be fitted with car black box so if militants had attacked or damaged the vehicle immediate SMS is send to military based station and this ammunitions can be made save from wrong hands.

\section{Conclusion}

The development of Event Data Recorder system based on ARM for Vehicle Monitor. The proper designed extreme conditions require getting data recorded into Event Data Recorder. This project aims to implement Event Data Recorder fault protection mechanism for vehicle to get data recorded and get alert message on LCD. In this project, sensing data, detection of extreme conditions and recording the respected conditional data into the SD card.

The system is an integrated platform using the GPS technology and other sensors to monitor cargo vehicles, but also be applied to other moving objects such as cars, boats, motorcycles, personnel, etc. provide location-based services, operational services, and management services as well as alarm monitoring for regulatory authorities. 
The developed system is a simple solution for surveillance, monitoring, security. The GPS provide accuracy less than $3 \mathrm{~m}$. They primarily refer to increasing the reliability of the end system with respect to prototype. It achieved the information exchange between monitoring centre and vehicle terminal.

The rapid storage, the real time data processor has the ability to read and write with high speed, which is the requirement of changing real-time data and high data sampling. In Large capacity, the storage system needs enough capacity to store the data so that it can be analyzed after an accident. The data in the Car Black Box should maintain and completely in a long term without any damage and lost.

\section{Future Scope}

It is expected that the very encouraging outcome of the project would encourage further development in this promising area. Thus it's leading to efficient commercial as well as industrial application. The future work will be to monitoring to the each module connected to the system i.e. as it working properly and also simultaneously video record of that extreme condition.

The Event Data Recorder is the most robust of the existing hardware devices. As the project implementation is done on a standalone system, forming a network of such system with the help of GPS module and sensors. Increase the utility of the system to a greater extent providing it a global connectivity. This may incorporate many useful features like position of vehicle and speed of vehicle. If the android or other application can be made the device can be very effectively used. Also use of TCP/IP protocol is very efficient for further development of project.

\section{Reference}

[1]. Yoshiyuki Tanaka, Naoki Yamada, Toshio Tsuji, Takamasa Suetomi, "Vehicle Active Steering Control System Based on Human Mechanical Impedance Properties of the Arms" IET International transport system conference 2014

[2]. Bing-Fei Wu, Ying-Han Chen, Chung-Hsuan Yeh, "Driving behaviour based event data recorder" IET International transport system conference 2013 ,pp.361-367.

[3]. Saurabh S. Chakole, Asstt. Prof. Vivek R. Kapur, Asst. Prof. Y. A. Suryawanshi, "ARM Hardware Platform for Vehicular Monitoring and Tracking”, 2013 International Conference on Communication Systems and Network Technologies, Nagpur, India, page no.757-761.

[4]. Yu-Kung Chen,Tsung Hsien, ” Sky recognisation for Driving-view images”, National science council. 2013 pp. 4799-3080

[5]. Chetan Patil, Yashwant Marathe, Kiran Amoghimath, Suman David S, "Low cost black box for cars" National institute of technology Karnataka Surathkal, Texas instruments India Educator's conference 2013 pp. 463-474

[6]. T.W. Chim, S.M. Yiu, C.Y. Yeung, Vicler O.K.Li \& Lucus C.K. Hu, "Securing, privacy-preserving, distributed Motor vehicle event data recorder," International conference on connected vehicle \& Expo in 2013 pp. 491-492

[7]. Duy Le Nguyen,Myung Eui Lee, Arteem Lensky, "The design and implementation of new vehicle black box using OBD information," in IEEE WCNC, 2013, pp. 506-511.

[8]. Tao Zhang, Airong Huang, Sishan Wang, "Design and Implementation of Vehicle Travelling Data Recorder Based on the Newest Chinese Standard", International Conference on Computational and Information Sciences,2013, pp. 1190-1193

[9]. Mooseop Kim and Chi Yoon Jeong, "An Efficient Data Integrity Scheme for Preventing Falsification of Car Black Box ", ICTC 2013, pp. 1020-1021

[10]. Artis Mednis y, Atis Elsts y, Leo Selavo y "Embedded Solution for Road Condition Monitoring Using Vehicular Sensor Networks", IEEE ,2012.

[11]. Zhang Wen, Jiang Meng” Design of Vehicle positioning System Based on ARM", Business Management and Electronic Information (BMEI),International Conference 2011 IEEE. 